\title{
Multidimensional Analysis of Copper Ore Flotation in Terms of Applied Hydrophobizing Agents
}

\author{
Paulina Pięta ${ }^{1, *}$, Tomasz Niedoba ${ }^{1}$, and Agnieszka Surowiak ${ }^{1}$ \\ ${ }^{1}$ AGH - University of Science and Technology, Faculty of Mining and Geoengineering, Department \\ of Environmental Engineering and Mineral Processing, Cracow, Poland
}

\begin{abstract}
Flotation is a method of enrichment used to distribute particles, which differ in their surface properties. Hydrophobic solids intrinsically create contact at the solid-liquid-gas interface. However, not all minerals, including copper minerals, can be characterized by this crucial ability. In that case it is necessary to use the collector reagents which guarantees a high efficiency of the enrichment process. The main aim of the paper was to examine the impact of selected collector types and dosages on the results of Polish sandstone copper ore flotation and to find optimal parameter values for products that meet quality and quantity requirements. The laboratory tests were carried out with an application of two types of collectors (Hostaflot, sodium ethyl xanthate aqueous solution) in dosages 100 and $150 \mathrm{~g} / \mathrm{Mg}$. Data analysis was based on the use of the taxonomy methods in order to select optimal conditions of collector dosage and type. Based on the indexes, it was found that the best enrichment effects were obtained with a sodium ethyl xanthate aqueous solution $150 \mathrm{~g} / \mathrm{Mg}$. Key words - flotation, copper ore, taxonomy methods, multidimensional comparative analysis.
\end{abstract}

\section{Introduction}

Flotation is a separation method used to separate particles which can be characterized by differences in surface properties. In a single flotation act of hydrophobic grains, a naturally occurring aggregate with an air bubble is formed and subsequently it floats to the froth. Hydrophobic solids intrinsically create contact at the solid-liquid-gas interface. However, not all minerals, including copper minerals, exhibit this key hydrophobic ability. The endurance of the occurred mineral-air bubble aggregate in the flotation pulp can be modified by the addition of suitable reagents. For hydrophilic particles, the formation of a three-phase system depends primarily on the addition of a hydrophobic reagent. The dosed reagent type may affect both the change in hydrophobicity and the final effect of flotation in the form of content and recovery of the metal in the separation products [3, 4].

A group of reagents that reduce the surface tension of a solid is broad. In general, ion collectors and nonionic collectors can be distinguished.

* Corresponding author: ppieta@agh.edu.pl 
The use of these reagents is conditioned by the properties of the minerals and guarantees a high efficiency of the enrichment process. Hence it is important to analyse their impact on the quality of the flotation products thoroughly. The application of chemical reagents is not the only significant factor affecting the final flotation effect. As indicated works $[14,15]$ the particle size distribution of feed material also plays a key role in the process. Properly selected communition and sizing processes provide a high level of copper minerals liberation, which guarantees better enrichment effects.

\section{Scope and Methodology}

The copper ores in LGOM are characterized by variable lithological composition, mineralization and floatability. The deposit is formed by an accumulation of copper sulphates occurring in sandstone, copper-bearing shales and carbonate rock. They are different in terms of physical and chemical properties and additionally they are varied locally within one type. Polish copper ores are dominated by copper sulphide minerals (chalcocite, bornite, chalcopyrite, cobalt). A major problem during the flotation process is the presence of a specific substance which is characterized by natural hydrophobicity, which gets across the concentrates and thus it deteriorates its quality. Even despite introduced collectors and frothers, it is necessary to use a multi-stage flotation process to achieve the desired results $[4,5,12]$.

The circuit comprises of rougher and main flotation operations, with a section of the ore carbonate and sandstone-shale. The products obtained in the first stages are directed downstream to the cleaning flotation process to achieve concentrate having the highest possible concentrate grade. The sodium ethyl xanthate aqueous solution or its mixture with isobutyl xanthate $(70 \mathrm{~g} / \mathrm{Mg})$ are used as collectors. Apart from the flotation reagents, the effects of copper ore enrichment depend on many factors which leads to the process being complicated and demanding. $[4,5]$.

\subsection{Multidimensional comparative analysis (MCA)}

The multidimensional structure of processes and studied objects, the complexity of the relationship between different properties characterize the whole reality surrounding us. Taking into account this condition is very important with regard to an optimization of processes and complete characterization of mineral resources $[4,11]$. Different methods of multidimensional comparative analysis serve as common tools used in economics, social studies, biological, anthropogenic and geographic researches. In the works $[1,6,7]$ taxonomic methods were successfully used to consider the issues of mineral processing. The undisputed advantage of these methods is their versatility, the simplicity of calculation and the ease of interpretation. They allow to replace the description of a multicharacteristic object in question with a synthetic variable which replaces all dependent variables with one value.

MCA is a comparison of objects defined with many diverse features. The methods to perform such an analysis include taxonomic methods, based on comparisons using the distance matrix. The $\boldsymbol{X}$ matrix (1) of diagnostic features is the basis for calculations and further analysis. Based on this, a synthetic matrix $\boldsymbol{Z}$ (2) of standardized values is calculated, ordering all nsituations in a linear manner, considering both positive (stimulant) and negative (destymulant) effects of the features on the phenomenon. 


$$
\begin{aligned}
\boldsymbol{X} & =\left[\begin{array}{llll}
x_{11} & x_{12} & \cdots & x_{1 l} \\
x_{21} & x_{21} & \cdots & x_{2 l} \\
\cdots & \cdots & \cdots & \cdots \\
\cdots & \cdots & \cdots & \cdots \\
x_{n 1} & x_{n 2} & \cdots & x_{n l}
\end{array}\right] \\
\boldsymbol{Z} & =\left[\begin{array}{llll}
z_{11} & z_{12} & \cdots & z_{1 l} \\
z_{21} & z_{21} & \cdots & z_{2 l} \\
\cdots & \cdots & \cdots & \cdots \\
\cdots & \cdots & \cdots & \cdots \\
z_{n 1} & z_{n 2} & \cdots & z_{n l}
\end{array}\right]
\end{aligned}
$$

where:

$x_{i j}-$ the value of a property $i$-number for test $j$-number;

$n$ - number of examined cases, i.e. flotation tests, $j=1, \ldots, n$;

$l$ - number of random variable, i.e. copper content, copper recovery, $i=1, \ldots, l$;

$z_{i j}-$ unitarized value of $x_{i j}$ property, which were calculated according to formula:

$$
z_{i}=\frac{x_{i}}{x_{\max }-x_{\min }},
$$

$x_{\max }, x_{\min }-$ the maximum and minimum value of the property.

Three methods of MCA were introduced to investigate the effects of sandstone copper ore flotation process. The taxonomic development measure with the Euclidean distance $d_{j}(3)$, the relative development index $w_{j}(4)$, and the zero-unification method $z_{j}(5)$ were used. A detailed description of the taxonomic measure can be found in $[6-8,10]$ below.

$$
\begin{gathered}
d j=\sqrt{\sum_{i=1}^{n}\left(1-s_{j i}\right)^{2}}, \\
w_{j}=\frac{\sum_{j=1}^{k} u_{i j}}{\sum_{j=1}^{k} \max _{j}\left\{u_{i j}\right\}}, \\
z_{j}=\frac{1}{n} \sum_{i=1}^{n} H_{i j},
\end{gathered}
$$

where:

$$
\begin{aligned}
& s_{j i}=\frac{z_{j i}}{z_{j \max }}, \\
& u_{i j}=x_{i j}^{*}+\left|\max _{i}\left\{x_{i j}^{*}\right\}\right|, \\
& x_{i j}^{*}=\frac{x_{i j}-\bar{x}_{i}}{S_{i}},
\end{aligned}
$$




$$
\begin{aligned}
& H_{i j}=\frac{x_{i j}-x_{i \text { min }}}{x_{i \max }-x_{i \min }} \text { for stymulants, } \\
& H_{i j}=\frac{x_{i \max }-x_{i j}}{x_{i \max }-x_{i \min }} \text { for destymulants, } \\
& S_{i}-\text { standard deviation of features } i \text {-number, } \\
& \bar{x}_{i}-\text { mean value of the feature } i \text {-number. }
\end{aligned}
$$

\section{Experimental}

The paper presents the results of research on the influence of selected types and dosages of collectors on the effects of flotation of sandstone copper ore. Laboratory tests were performed using two anionic collectors: sodium ethyl xanthate aqueous solution and Hostaflot. They were added in two dosages: 100 and $150 \mathrm{~g} / \mathrm{Mg}$. Nasfroth in dosage $50 \mathrm{~g} / \mathrm{Mg}$ was used as a frother during the test.

Selected taxonomic methods were used to analyse the experimental data. A multidimensional comparative analysis has allowed us to assess the effectiveness of the sandstone copper ore flotation process and to classify the case studies.

The testing material was a sample of Polish copper ore which was taken from ZG Polkowice KGHM Polska Miedź S.A. For flotation tests a sandstone type was used, which was previously separated from the raw material and then it was crushed and classified to obtain a 40-71 $\mu \mathrm{m}$ size fraction. The copper content in sandstone type was equal to $1.1 \%$. The tests were carried out in a Denver flotation machine with a constant concentration flotation slurry of $200 \mathrm{~g} / \mathrm{dm}^{3}$, aerating and at a rotor speed. The research was carried out while maintaining uniform material preparation procedures. During a single experimental study one concentrate and tailings were obtained. The flotation products were dried and weighed and subjected to X-ray fluorescence to determine the copper content. For each experiment, the following process evaluation indicators were identified: copper content in concentrate (6), copper content in tailings (7), copper recovery in concentrate (8), copper recovery in tailings (9). The averaged values of selected flotation tests for the sandstone copper ore are presented in Table 1.

$$
\begin{gathered}
\beta_{i}=\frac{\sum_{i=1}^{n} \gamma_{i} \cdot \lambda_{i}}{\sum_{i=1}^{n} \gamma_{i}}, \\
\vartheta_{i}=\frac{\sum_{i=1}^{n} \gamma_{i} \cdot \lambda_{i}}{1-\sum_{i=1}^{n} \gamma_{i}}, \\
\varepsilon_{i}=\frac{\beta_{i}}{\alpha} \sum_{i=1}^{n} \gamma_{i}, \\
\eta_{i}=\frac{\vartheta_{i}}{\alpha} \sum_{i=1}^{n} \gamma_{i},
\end{gathered}
$$

where:

$\alpha-$ a copper content in the feed [\%], 
$\gamma_{i}=\frac{m_{i}}{\sum_{i=1}^{n} m_{i}} \times 100-$ a yield of a product $[\%]$,

$\lambda_{i}-$ content of copper in a product,

$m_{i}-$ an mass of a product.

Table 1. Results of flotation tests of sandstone lithological type for process duration equal to $30 \mathrm{~min}$

\begin{tabular}{|c|c|c|c|c|c|c|}
\hline $\begin{array}{c}\text { Size } \\
\text { fraction, } \\
{[\boldsymbol{\mu \mathbf { m } ]}}\end{array}$ & $\begin{array}{c}\text { Collector } \\
\text { type }\end{array}$ & $\begin{array}{c}\text { Collector } \\
\text { dosage, } \\
{[\mathbf{g} / \mathbf{M g}]}\end{array}$ & $\boldsymbol{\beta}_{\boldsymbol{i}}$ & $\boldsymbol{v}_{\boldsymbol{i}}$ & $\boldsymbol{\varepsilon}_{\boldsymbol{i}}$ & $\boldsymbol{\eta}_{\boldsymbol{i}}$ \\
\hline $125-200$ & $\mathrm{X}$ & 100 & 0,0215 & 0,0004 & 0,8000 & 0,2000 \\
\hline $125-200$ & $\mathrm{H}$ & 100 & 0,0270 & 0,0010 & 0,5813 & 0,4187 \\
\hline $125-200$ & $\mathrm{X}$ & 150 & 0,0264 & 0,0003 & 0,9177 & 0,0823 \\
\hline $125-200$ & $\mathrm{H}$ & 150 & 0,0799 & 0,0003 & 0,9018 & 0,0982 \\
\hline $100-125$ & $\mathrm{X}$ & 100 & 0,0678 & 0,0005 & 0,8704 & 0,1296 \\
\hline $100-125$ & $\mathrm{H}$ & 100 & 0,0326 & 0,0014 & 0,5933 & 0,4067 \\
\hline $100-125$ & $\mathrm{X}$ & 150 & 0,0712 & 0,0002 & 0,9429 & 0,0571 \\
\hline $100-125$ & $\mathrm{H}$ & 150 & 0,0688 & 0,0003 & 0,9396 & 0,0604 \\
\hline $71-100$ & $\mathrm{X}$ & 100 & 0,0455 & 0,0003 & 0,9539 & 0,0461 \\
\hline $71-100$ & $\mathrm{H}$ & 100 & 0,0314 & 0,0016 & 0,6983 & 0,3017 \\
\hline $71-100$ & $\mathrm{X}$ & 150 & 0,0486 & 0,0002 & 0,9623 & 0,0377 \\
\hline $71-100$ & $\mathrm{H}$ & 150 & 0,0522 & 0,0004 & 0,9398 & 0,0602 \\
\hline $40-71$ & $\mathrm{X}$ & 100 & 0,1467 & 0,0005 & 0,9669 & 0,0331 \\
\hline $40-71$ & $\mathrm{H}$ & 100 & 0,5134 & 0,0003 & 0,9808 & 0,0192 \\
\hline $40-71$ & $\mathrm{X}$ & 150 & 0,0298 & 0,0001 & 0,9943 & 0,0057 \\
\hline $40-71$ & $\mathrm{H}$ & 150 & 0,0344 & 0,0002 & 0,9410 & 0,0590 \\
\hline $20-40$ & $\mathrm{X}$ & 100 & 0,0428 & 0,0004 & 0,9505 & 0,0495 \\
\hline $20-40$ & $\mathrm{H}$ & 100 & 0,1585 & 0,0007 & 0,9708 & 0,0292 \\
\hline $20-40$ & $\mathrm{X}$ & 150 & 0,0347 & 0,0003 & 0,9711 & 0,0289 \\
\hline $20-40$ & $\mathrm{H}$ & 150 & 0,0300 & 0,0004 & 0,9011 & 0,0989 \\
\hline $0-20$ & $\mathrm{X}$ & 100 & 0,0843 & 0,0009 & 0,9768 & 0,0232 \\
\hline $0-20$ & $\mathrm{H}$ & 100 & 0,0947 & 0,0012 & 0,9625 & 0,0375 \\
\hline $0-20$ & $\mathrm{X}$ & 150 & 0,0426 & 0,0007 & 0,9534 & 0,0466 \\
\hline $0-20$ & $\mathrm{H}$ & 150 & 0,0479 & 0,0007 & 0,9762 & 0,0238 \\
\hline
\end{tabular}

symbols: H-collector of the izobutyl ksanthate family Hostaflot, X-collector of the ethyl ksanthate family

\section{Analysis results}

The assessment of the efficiency of copper ore enrichment was based on the following indicators: content and recovery of metal in concentrate and tailings. The results of laboratory experiments have confirmed that the highest quality of the concentrates was observed when a feed was a 40-71 $\mu \mathrm{m}$ size fraction. The optimal particle size distribution for this lithological type was based on the $d_{j}$ values, which take into account $\beta, v, \varepsilon, \eta$ for the assessment process (Tab. 3). The further part of the analysis focused on that material. In the case of a single flotation test duration assortment, such unambiguous results were not obtained. Hence the results of the flotation after 1, 12, 30 [min] were presented and compared. 
The effectiveness of the flotation process was assessed on the basis of two groups of indicators characterizing positive $(\beta, \varepsilon)$ and negative $(v, \eta)$ enrichment results. To consider, the direction of influence of the variables in the MCA, it was necessary to replace the value of the copper content and the recovery of this metal in the tailings by their opposite. Thus, a matrix of variable values for which a homogeneous final evaluation of the results could be made.

Another important issue is normalization, which unifies the values of features from the point of view of a certain criterion [2]. The main goal of this kind of transformation is to fulfill the postulate of additivity, i.e. to bring features with different denomination to their comparability $[2,8-10,13]$. In the calculation process, two forms of standardization were chosen (a quotient conversion $s_{j i}$ and standardization $x_{i j}{ }^{*}$ ).

Taking into account the number of variables, the taxonomy measures were described by the following formulas (10-12):

- Euclidean distance,

$$
d_{j}=\sqrt{\left(1-\frac{z_{\beta i}}{z_{\beta \max }}\right)^{2}+\left(1-\frac{z_{\vartheta i}}{z_{\vartheta \max }}\right)^{2}+\left(1-\frac{z_{\varepsilon i}}{z_{9 \max }}\right)^{2}+\left(1-\frac{z_{\eta i}}{z_{\eta \max }}\right)^{2}},
$$

- relative development index

$$
w_{j}=\frac{u_{\beta j}+u_{9 j}+u_{\varepsilon j}+u_{\eta j}}{\max _{i}\left\{u_{\beta j}\right\}+\max _{i}\left\{u_{\vartheta_{j}}\right\}+\max _{i}\left\{u_{\varepsilon j}\right\}+\max _{i}\left\{u_{\eta j}\right\}},
$$

where:

$$
\begin{aligned}
& u_{\beta j}=x_{\beta j}{ }^{*}+\left|\max _{i}\left\{x_{\beta j}^{*}\right\}\right|, \quad u_{9 j}=x_{9 j}{ }^{*}+\left|\max _{i}\left\{x_{9 j}{ }^{*}\right\}\right|, \\
& u_{\varepsilon j}=x_{\varepsilon j}{ }^{*}+\left|\max _{i}\left\{x_{\varepsilon j}^{*}\right\}\right|, \quad u_{\eta j}=x_{\eta j}{ }^{*}+\left|\max _{i}\left\{x_{\eta j}{ }^{*}\right\}\right|,
\end{aligned}
$$

- zero-unification method

$$
z_{j}=\frac{1}{n} \cdot\left(\frac{x_{\beta j}-x_{\beta \min }}{x_{\beta \max }-x_{\beta \min }}+\frac{x_{\varepsilon j}-x_{\varepsilon \min }}{x_{\varepsilon \max }-x_{\varepsilon \min }}+\frac{x_{\vartheta_{\max }}-x_{\vartheta j}}{x_{\vartheta \max }-x_{\vartheta \min }}+\frac{x_{\eta \max }-x_{\eta j}}{x_{\eta \text { max }}-x_{\eta \min }}\right)
$$

Based on the results of the taxonomic measure determined for a single flotation test it was possible to compare the level of the studied phenomenon. Euclidean distances are meters that use a pattern which is the maximum value of a feature. This is due to the fact that a higher value of the indicator is equivalent to a worse effect of the sandstone copper ore flotation. The reverse situation exists for the (relative development index, zero unification method), which are not included in the calculation process of the pattern. The values of indexes $w_{j}$ and $z_{j}$ are from $0-1$. As the result is closer to 1 , this phenomenon is better in terms of the general criterion. Table 2 summarizes the results of the overall evaluation of sandstone copper ore flotation under defined conditions for all three types of taxonomic methods.

The results of the MCA indicated that the best results of flotation were achieved with $150 \mathrm{~g} / \mathrm{Mg}$ ethyl xanthate and a extended process time up to 30 minutes. This conclusion was confirmed by each taxonomic method. As the process duration was shortened, the quality of the flotation concentrates deteriorated and the optimal process conditions changed. It can be seen that the best quality of the separation products during the 12-minute working time provided a Hostaflot reagent dosed at $100 \mathrm{~g} / \mathrm{Mg}$. The evaluation for the shortest time of the separation 
process did not produce consistent results, as in the other cases. Two out of three indices have shown that the process is best carried out with the use of ethyl xanthate in $100 \mathrm{~g} / \mathrm{Mg}$.

Table 2. The evaluation of the effectiveness of sandstone copper ore flotation process including three taxonomic measures

\begin{tabular}{|c|c|c|c|c|c|}
\hline $\begin{array}{c}\text { Collector } \\
\text { type }\end{array}$ & $\begin{array}{c}\text { Collector } \\
\text { dosage, }[\mathrm{g} / \mathrm{Mg}]\end{array}$ & $\begin{array}{l}\text { Flotation } \\
\text { time, [min] }\end{array}$ & $d_{j}$ & $z_{j}$ & $w_{j}$ \\
\hline $\mathrm{X}$ & 100 & \multirow{4}{*}{1} & $1,4868^{*}$ & 0,5440 & $0,2846 *$ \\
\hline $\mathrm{H}$ & 100 & & 1,5172 & 0,4566 & 0,2529 \\
\hline $\mathrm{X}$ & 150 & & 1,6778 & $0,5799 *$ & 0,1814 \\
\hline $\mathrm{H}$ & 150 & & 1,7239 & 0,1295 & 0,0119 \\
\hline $\mathrm{X}$ & 100 & \multirow{4}{*}{12} & 1,5863 & 0,6398 & 0,2632 \\
\hline $\mathrm{H}$ & 100 & & $1,4698 *$ & $0,7924 *$ & 0,3376* \\
\hline$X$ & 150 & & 1,7062 & 0,7344 & 0,2391 \\
\hline $\mathrm{H}$ & 150 & & 1,7024 & 0,5923 & 0,1906 \\
\hline$X$ & 100 & \multirow{4}{*}{30} & 1,5845 & 0,6807 & 0,2721 \\
\hline $\mathrm{H}$ & 100 & & 1,4141 & 0,7579 & 0,4216 \\
\hline$X$ & 150 & & 0,9419* & 0,9218* & $0,8147^{*}$ \\
\hline $\mathrm{H}$ & 150 & & 1,6950 & 0,6434 & 0,2123 \\
\hline
\end{tabular}

highlighted values indicate the best course of the flotation process

** symbols: H-collector of the izobutyl ksanthate family Hostaflot, X-collector of the ethyl ksanthate family

Table 3. The assessment of flotation results for size fractions

\begin{tabular}{|c|c|}
\hline Size fraction, $[\boldsymbol{\mu m}]$ & $\boldsymbol{d}_{\boldsymbol{j}}$ \\
\hline $40-71$ & 1,329741 \\
\hline $100-125$ & 1,640433 \\
\hline $71-100$ & 1,661561 \\
\hline $20-40$ & 1,678242 \\
\hline $0-20$ & 1,679803 \\
\hline $125-200$ & 1,689951 \\
\hline
\end{tabular}

Based on the results of the MCA conducted by means of different methods, it can be stated that both the Euclidean distance and the relative development index allow to draw the same conclusions. Certain differences were observed only in one case, using zero unification method to evaluate the overall phenomenon.

\section{Conclusions}

Undoubtedly, such factors as the type and dosage of the collecting reagent constitute very important features affecting the efficiency of copper ore flotation. This is due to the lack of natural hydrophobicity of copper minerals, which, under natural conditions, are unable to produce durable aggregates of air-bubbles to be floated into a froth.

The results of the sandstone copper ore flotation test were analyzed for four variables: copper content in concentrate, copper content in tailings, copper recovery in concentrate, copper recovery in tailings. By analyzing the raw experimental data, we could see the apparent differences between the values of $\beta, \varepsilon, \eta$ in the individual products of the partition which provided the basis for the preliminary conclusions. Despite the differences in the dosage and type of collector, the $v$ remained at a similar level. Minor fluctuations of the metal content in 
the tailings significantly affect the efficiency of the whole copper ore enrichment. A complete evaluation of the variables was performed using three taxonomic methods $\left(d_{j}, w_{j}, z_{j}\right)$ to compare the results for the variable dosages and types of collectors. Based on the taxonomic measures, it was found that the best enrichment effects were obtained with $150 \mathrm{~g} / \mathrm{Mg}$ sodium ethyl xanthate aqueous solution at extended flotation time $(30 \mathrm{~min})$. The results obtained in three ways gave consistent conclusions, despite various computational techniques and approaches to the analysis of the phenomenon.

\section{Acknowledgements}

The paper is the effect of the scientific project no. 15.11.100.021.

\section{References}

1. C. Aldrich, Exploratory analysis of metallurgical process data with neural networks and related methods. Process Metallurgy 12. Elsevier Science B.V., Netherlands (2002)

2. T. Borys, Elements of quality theory. PWN, Warsaw (1980)

3. J. Drzymała, Mineral processing. Foundations of theory and practice of minerallurgy. Oficyna Wydawnicza PWr, Wroclaw (2007)

4. M. Duchnowska, E. Kasińska-Pilut, A. Bakalarz, K. Konieczny, P. Kowalczuk, A. Luszczkiewicz, Multivariate statistical analysis of upgrading copper ore in ZWR Polkowice. CUPRUM, 2(75), 97-108 (2015)

5. D. Foszcz, Results of determining the optimal results of multicomponent copper ores beneficiation. Mineral Resources Management, Cracow (2013)

6. D.W. Ginsberg, W.J. Whiten, Cluster analysis for mineral processing applications. Trans. Instn. Min. Metall., 100, 139-146 (1991)

7. D.W. Ginsberg, W.J. Whiten, Expert system development using ellipsoid-based clustering. Minerals Engineering, 6(1), 31-40 (1993)

8. M. Jarocka, The choice of a formula of the data normalization in the comparative analysis of multivariate objects. Economics and Management, 1, 113-126 (2015)

9. J. Kurkiewicz, J. Pociecha, K. Zając, Methods of multidimensional comparative analysis in demographic development research. SGH ISiD, Monograph 336, Warsaw (1991)

10. M. Łuniewska, W. Tarczyński, Methods of multidimensional comparative analysis on the capital market. PWN, Warsaw (2006)

11. T. Niedoba, Multidimensional characteristics of random variables in description of grained materials and their separation processes. Mineral Resources Management, Cracow (2013)

12. M. Nieć, A. Piestrzyński, Monograph KGHM PM SA II. 2.19 Mineralisation. KGHM CUPRUM Sp. z o.o. CBR, Wroclaw (2007)

13. B. Pawełek, Methods of normalization of variables in comparative studies of complex economic phenomena. UE, Cracow (2008)

14. A. Potulska, An influence of fine grinding operations on flotation effectiveness of domestic copper ores. Doctoral thesis; Politechnika Wrocławska, Wydział Geoinżynierii, Górnictwa i Geologii, Wrocław (2008)

15. D. Saramak, A. Krawczykowska, A. Młynarczykowska, Effects of high pressure ore grinding on the efficiency of flotation operations. AMS, 59(3), 730-741 (2014) 